\title{
MANTLE DYNAMICS WITH INDUCED PLATE TECTONICS
}

\author{
Yanick Ricard and Christophe Vigny
}

Département de Géologie, Ecole Normale Supérieure, Paris

Abstract. Mantle circulation and plate tectonics can be described by means of two scalar fields. The poloidal field is related to horizontal divergence, i.e., to the vertical mass flux. The toroidal field expresses the shear deformation or the rotations within spherical shells. For the Earth, the surface kinetic energy is known to be equally distributed between the two fields. In recent years the lateral mantle density variations revealed by seismic tomography have been introduced in dynamic mantle models which have predicted the major features of both the geoid and the surface divergence. These models assumed a spherical symmetry of the rheological properties of the Earth. However, with this assumption, the Navier-Stokes equation implies that mass heterogeneities cannot drive any toroidal motion. This prompted us to develop a new model which takes into account the existence of rigid and independent plates. These plates break the spherical symmetry assumed in all earlier models. They modify the mantle circulation and hence the predicted surface observables such as displacement and gravity. In this paper, we first explain by means of a very simple twoplate model, how the poloidal flow caused by mantle heterogeneities can be converted to surface toroidal motion. We then apply the model to the real Earth with its major plates and compare our predictions to those deduced from a more classical Earth model having a uniform free slip condition at the surface. Taking the seismic tomography pattern as a picture of the mantle mass heterogeneities, we achieve a highly satisfactory fit to the observed plate velocities. This new theoretical framework also improves the geoid prediction.

\section{Introduction}

In the last few years, the complex relationships between deep mass heterogeneities and surface observables, such as topography, horizontal surface velocities, and gravity, have been derived by various authors [Kaula, 1975; Hager and 0'Connell, 1979; Ricard et al., 1984; Richards and Hager, 1985; Forte and Peltier, 1987]. In most of these studies the real Earth is modelled as a self-gravitational viscous sphere. The viscosity is assumed to be Newtonian and only varies with depth. The lateral density variations within the mantle induce a flow which deflects the various interfaces such as the Earth's surface and the core-mantle boundary. The gravity field depends both on the internal masses and on the shapes of the dynamically perturbed interfaces.

Copyright 1989 by the American Geophysical Union.

Paper number $89 \mathrm{JB} 01292$.

0148-0227/89/89JB-01292\$05.00
The predictions of these models have been test against observations. Using a realistic mass distribution for slabs and assuming that seismic tomography reflects the deep mantle structure, satisfactory geoid configuration and surface velocity divergence have been computed [Hager et al., 1985: Forte and Peltier, 1987; Ricard et al., 1988].

The physical equations which have been widely used to model the large-scale dynamic behavior of the Earth are the Navier-Stokes, Poisson, and mass conservation equations which read

$$
\begin{gathered}
\eta \vec{\nabla}^{2} \overrightarrow{\mathrm{V}}=\vec{\nabla} \mathrm{p}-\rho \vec{\nabla} \mathrm{U}-\delta \rho \overrightarrow{\mathrm{g}} \\
\nabla^{2} \mathrm{U}=-4 \pi G \delta \rho \\
\vec{\nabla} \overrightarrow{\mathrm{V}}=0
\end{gathered}
$$

In these expressions, the velocity field $\vec{V}$ is induced by the nonhydrostatic pressure $p$ and by the lateral density variations $\delta \rho$. The perturbed gravity potential $U$ due to the density variations $\delta \rho$ appears in equation (1) describing the dynamic behaviour because of the self-gravitational effect. The viscosity $\eta$ and the density $\rho$ are assumed to be uniform, and $G$ is the gravitational constant. By inspection of equation (3), one sees that the velocity $\vec{V}$ is the curl of a vector field and can be written in the most general way on the form:

$$
\overrightarrow{\mathrm{v}}=\vec{\nabla} \otimes \mathrm{Te}_{1}+\vec{\nabla} \otimes \vec{\nabla} \otimes \mathrm{Se}_{1}
$$

The scalar functions $S$ and $T$ are the poloidal and toroidal components of the flow, and $\vec{e}_{1}$ is the unit radial vector. The physical meaning of these new fields is easily understood. It can be shown that the horizontal divergence $\vec{\nabla}_{H} \overrightarrow{\vec{V}}$, which is the divergence of the horizontal component of the velocity $\vec{V}$ is related only to the poloidal component, whereas the radial vorticity $(\vec{\nabla} \otimes \vec{v}) \vec{e}_{1}$ depends only upon the toroidal component. The relationships between these fields read

$$
\begin{gathered}
\vec{\nabla} \vec{V}=\frac{1}{r^{2}} \frac{\partial}{\partial r}\left(r^{2} \nabla_{H}^{2} \mathrm{~S}\right) \\
(\vec{\nabla} \otimes \vec{V}) \vec{e}_{1}=-\nabla_{H}^{2} T
\end{gathered}
$$

where $\nabla_{H}^{2}$ is the horizontal Laplace operator. At the Earth's surface, the converging zones of subduction or collision and the diverging ridges express the existence of internal poloidal flow. On the other hand, the strike-slip zones reveal the vigor of the toroidal component. Taking the curl of the Navier-Stokes equation (1) and expressing $\vec{V}$ as a function of $S$ and $T$ following equation (4), one can derive two new equations which decouple the poloidal and toroidal fields by 


$$
\begin{gathered}
\nabla^{4} \mathrm{~S}=\frac{\delta \rho \mathrm{g}}{\eta} \\
\nabla^{2} \mathrm{~T}=0
\end{gathered}
$$

Thus the internal mass heterogeneities induce the poloidal field, but there are no excitation sources for the toroidal field. The latter should therefore vanish. For the real Earth, this is not the case. On the contrary, a quasi-equipartition of kinetic energy between the two modes is observed. For whole mantle problems, the global scale of the fields make the spectral representation in term of spherical harmonics appropriate. The power spectra of both the observed poloidal and toroidal surface fields vary like $l^{-2}$ [Hager and O'Connell, 1979; Forte and Peltier, 1987]. This striking observation obviously means that the theoretical framework given by equations (1). (2), and (3) is insufficient.

The only way to generate toroidal motion in a viscous fluid is to allow the viscosity to vary laterally. In this case the Navier-stokes equation (1) is not valid and must be replaced by the more general momentum equation (9) which reads

$$
\eta \vec{\nabla}^{2} \vec{V}+2 \vec{E} \vec{\nabla} \eta=\vec{\nabla} p-\rho \vec{\nabla} U-\delta \rho \vec{g}
$$

Here $\vec{E}$ is the strain rate tensor, and $\eta$ is the viscosity which can vary spatially within the fluid.

For simplicity let us neglect the Earth's sphericity and assume that the gravity $\overrightarrow{\mathrm{g}}$ is parallel to the vertical unit vector $\vec{z}$. To infer the generation of toroidal motion, let us again take the curl of equation (9) and project the resulting equation onto the vertical vector. After a somewhat cumbersome algebra, one finds

$$
\eta \nabla^{2} \xi+\vec{\nabla} \xi \vec{\nabla} \eta=-\frac{1}{\eta}(\vec{\nabla} \eta \otimes \vec{\nabla} p) \vec{z}
$$

where $\xi=(\vec{\nabla} \otimes \vec{V}) \vec{z}$ is the vertical vorticity. This last equation was given incorrectly in a paper by Kaula [1980] where it appeared without the term on the right hand-side. It is complex, but its physical meaning is clear. To drive the toroidal field, the Earth's dynamic behavior thus needs to produce a nonzero $(\vec{\nabla} \eta \otimes \vec{\nabla} p) \vec{z}$. In other words, lateral pressure variations must include a component orthogonal to lateral variations in viscosity. This also indicates that radial viscosity variations are inefficient in exciting toroidal motions. These conclusions remain true for a spherical Earth, but other components related to $\xi$ and its partial derivatives appear in equation (10). The steeper the viscosity transitions, the more vigorous the toroidal motion.

Long-wavelength viscosity variations are known to exist within the Earth. Some of them correlate with tectonic provinces like young oceans and old cratons. Their effects on global dynamic models have already been analyzed [Ricard et al., 1988]. More generally, lateral variations in viscosity within the deep convecting mantle can be related to temperature and thus be associated with density variations [Richards et al., 1989]. The general conclusion of the two papers just quoted is that a mass anomaly with a given wavelength can interact with the viscosity structure and generates surface observables which contains other wavelengths. These models with long-wavelength viscosity variations excite radial vorticity [Ricard et al., 1988], but the toroidal energy remains rather low in comparison with the poloidal energy. The energy equipartition between toroidal and poloidal fields seems to indicate that viscosity variations occur on a length scale smaller than the characteristic plate size. For the real Earth, the sharpest viscosity contrasts occur, of course, near plate boundaries. In what follows, we present a model which takes the very existence of plates into account. This model explains the generation of the toroidal flow and clearly demonstrates that surface plates strongly affect the whole mantle dynamics.

The Physical Model

Our model consists of a spherical self-gravitational viscous mantle overlaid by rigid plates and surrounding an inviscid core. The number and the shape of plates represent a priori data. In other words, we do not deal with the problem of their creation or evolution. Deep mantle heterogeneities produce a circulation which drives the overlaying plates. There are no direct interactions between plates, but the motion of a given plate partly controls the mantle flow and therby indirectly interacts with the others. Mechanical equilibrium requests that the torque of all applied forces on each plate be zero [Lliboutry, 1972; Forsyth and Uyeda, 1975]. Our mathematical approach consists in superposing two subsolutions. The first one is excited by imposed internal loads with a no-slip condition at the outer spherical surface. The second one has no internal loading and is excited by a horizontal surface velocity field described by an unknown set of rotation vectors. The values of these rotation vectors are deduced from the balance of the mechanical torques applied on each plate by the two subsolutions. For mathematical convenience the computation has been done using a generalized spherical harmonic representation truncated for degrees larger than 15. This formalism was introduced in seismological theory [Phinney and Burridge, 1973] and can readily be applied to dynamical problems. It enables one to handle simultaneously scalar, vectorial, and tensorial quantities, and it provides an elegant way of writing differential operators like gradient, divergence, or curl. The indices $n, l$, and $m$ refer to a representation on the basis of generalized spherical harmonics. In the mathematical appendix, the relationship between the components $v^{n} l_{m}$ of the surface velocity field and the horizontal shear stress components $\tau_{i n t}^{n} l_{m}$ generated by internal loads with a no-slip boundary condition at the surface is found to read (see equation (A18))

$$
v^{n l m}=\frac{R}{\eta_{0}} G^{*}\left(G K G^{*}\right)^{-1} G \tau_{i n t}^{n \cdot l \cdot m^{*}}
$$

Here $R$ is the Earth's radius, and $\eta_{0}$ is the reference viscosity. The matrix $K$ describes the linear relationship between imposed surface velocities $v^{n^{l m}}$ and the surface stresses $T_{e x t}^{n l m}$ that 


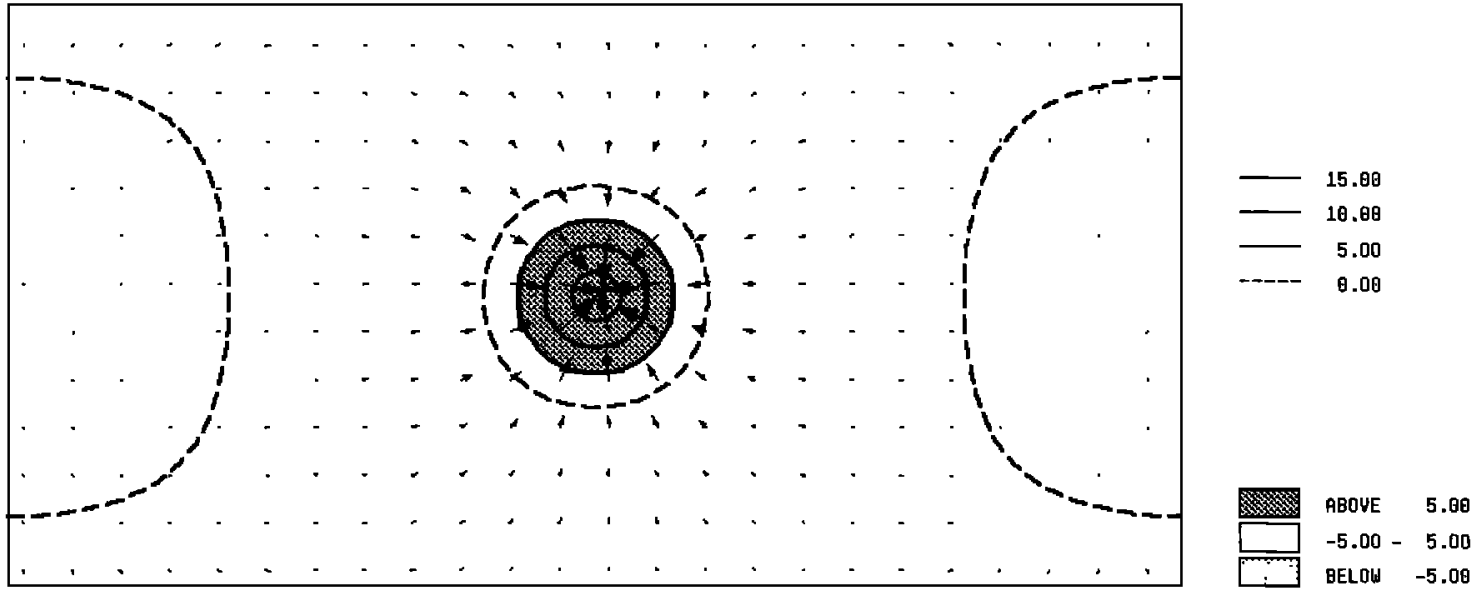

SCALE : $\rightarrow 0.30 \mathrm{CM} / \mathrm{YR}$.

Fig. 1. Computed geoid and surface velocity fields induced by a circular mantle mass anomaly located at a depth of $300 \mathrm{~km}$ and having the shape of a spherical cap of radius $1500 \mathrm{~km}$. The model Earth is mapped through a linear representation of both longitude and latitude. Its interior has a purely spherical symmetry and is made up of three layers: a lithosphere with viscosity $10^{22} \mathrm{~Pa} s$, an upper mantle with viscosity $10^{21} \mathrm{~Pa} \mathrm{~s}$, and a stiffer lower mantle with viscosity $10^{23} \mathrm{~Pa} \mathrm{~s}$. The computation was carried out up to the harmonic degree $l=15$. The level lines for the geoid are $5 \mathrm{~m}$ apart and the regions above $5 \mathrm{~m}$ are shaded. The surface velocity field radially converges toward the center of the anomaly and reaches a maximum of $0.3 \mathrm{~cm} / \mathrm{yr}$.

they generate in the absence of internal loads (see (A8))

$$
\tau_{\mathrm{e} \times t}^{\mathrm{n} l m}=\frac{\eta_{0}}{\mathrm{R}} \mathrm{K} \mathrm{v}^{\mathrm{n}} \cdot l \cdot m^{\prime}
$$

The operator $\mathrm{G}$ expresses the coupling between the induced plate kinematics and the internal circulation. In the absence of rigid surface plates, $G$ equals the identity matrix, and (11) reads simply

$$
\mathrm{v}^{\mathrm{n} m}=\frac{\mathrm{R}}{\eta_{0}} \mathrm{~K}^{-1} \quad \tau_{1 \mathrm{n} \mathrm{t}}^{\mathrm{n}} \cdot l \cdot m^{\prime}
$$

The general formalism which enables one to compute $K$ and $T_{i}^{n} l_{n}$, was fully described in recent papers [e.g.. Ricard et al., 1984]. It consists in computing Green's excitation functions for each spherical harmonic degree. A radial convolution of the Green's functions with the radial load distributions is then carried out for every degree $l$ and order $m$. The details for constructing the matrix $G$ are found in the mathematical appendix.

Our numerical code thus computes the pole and the rotation velocity of each plate caused by the internal load distribution within the mantle. Such a velocity pattern contains both poloidal and toroidal components. This is due to the imposed rigidity of plates which only allow deformation near their boundaries. The generation of toroidal motion is the major novelty of this paper. The global dynamics is drastically different from that of earlier Earth models with free slip or no-slip condition on the outer surface. The predicted topography and geoid will of course be affected by the presence of plates. In this new framework one should notice that whatever the wavelength of the internal load, it produces a velocity field containing all possible wavelengths. Nevertheless, numerical experiments have shown that the long-wavelength results are quite insensitive to the short-wavelength components of the loads.

\section{A Numerical Example}

To get some insight into the physics of our problem, let us take a very simple Earth model where the mantle is overlaid by two hemispheric shells of thicknesses $L=100 \mathrm{~km}$. The mantle flow is driven by a circular, horizontal mass heterogeneity of radius $R=1500 \mathrm{~km}$ at a depth $z=300$ $\mathrm{km}$. This load is located just below the great circle defining the boundary between the two plates. It has a prescribed surface density of $10^{6} \mathrm{~kg} / \mathrm{m}^{2}$, and therefore would correspond, assuming isostasy, to a surface topography of 350 $m$. The upper mantle viscosity is fixed at $10^{21} \mathrm{~Pa} \mathrm{~s}$, whereas three different viscosities, amounting to $10^{21}, 10^{22}$, and $10^{23} \mathrm{~Pa} \mathrm{~s}$, are considered for the lower mantle. The results of our models are compared to those produced by classical models with a uniform free slip condition at the surface. Both types of models, with and without plates, have the same internal viscosity profile except for the presence of a 100-km-thick lithosphere with viscosity $10^{22} \mathrm{~Pa} \mathrm{~s}$ in the first case.

First, we consider a model Earth with a uniform free slip condition at the surface and with a viscosity increase by a factor 100,10 , or 1 at $650 \mathrm{~km}$ depth. Figure 1 depicts the surface velocity field and the induced geoid for a viscosity ratio of 100 . The dense mass sinks within the mantle and induces a symmetrically converging 

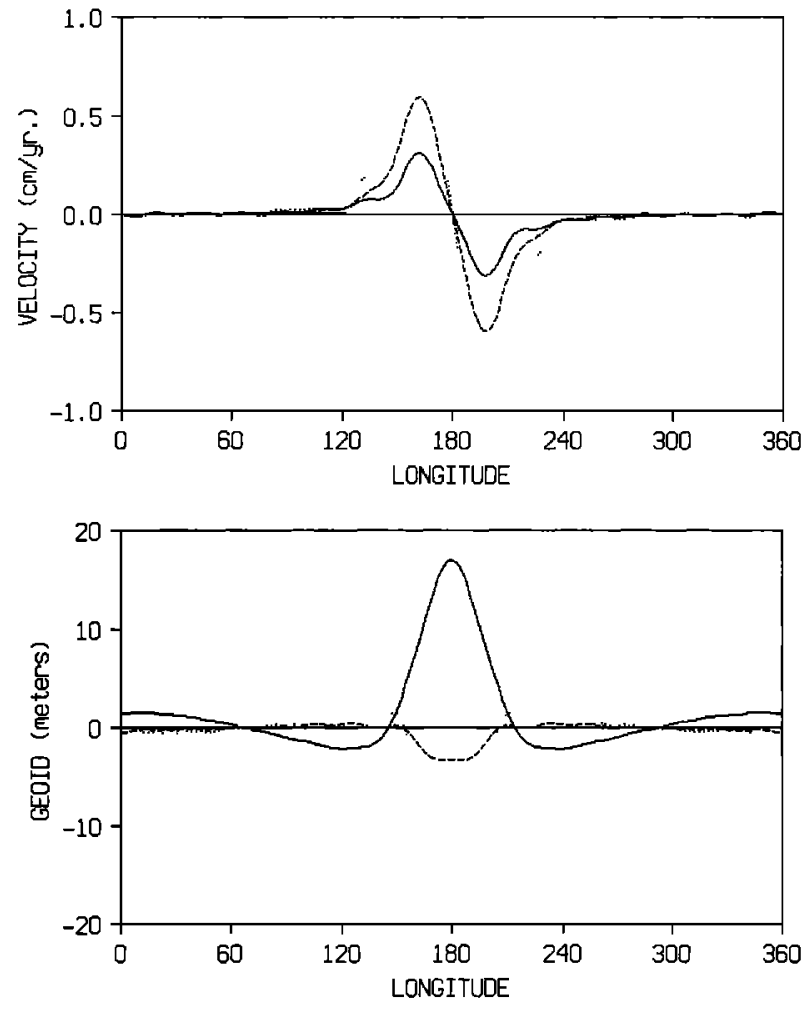

Fig. 2. Equatorial profiles of the geoid and the velocity. The solid curve corresponds to the section of Figure 1 where the viscosity of the lower mantle is 100 times that of the upper mantle. Two other computations for a viscosity jump reduced to a factor of 10 and for a uniform mantle led to the dashed and dotted profiles, respectively. For a model Earth with a purely radial viscosity and our chosen circular mass anomaly, the sections are the same across all great circles crossing the center of this mass. purely poloidal flow. The geoid exhibits a maximum located just above the deep heterogeneity which has the same symmetry as the load. Equatorial profiles of the velocity and the geoid are displayed in Figure 2 (top and bottom). The solid curves correspond to the equatorial section of the map depicted on Figure 1. The dashed curves stand for a viscosity jump by a factor 10 at 650 $\mathrm{km}$, and the dotted curves correspond to a uniform mantle. The velocities (Figure 2, top) are antisymmetrical and reach their extrema above the edge of the deep mass. The undulations are only due to Gibbs' effect related to our truncation degree $l=15$. The maximum velocity value increases as the lower mantle viscosity decreases, and it reaches $0.9 \mathrm{~cm} / \mathrm{yr}$ for a uniform mantle. The sign of the induced geoid (bottom) changes according to the value of the viscosity jump at the upper-lower mantle interface. This result is well known [Ricard et al., 1984; Hager, 1984; Lago and Rabinowicz, 1984]. Indeed, in a mantle with uniform viscosity, the dense mass deflects the lithosphere downward by $332 \mathrm{~m}$, somewhat less than for 1sostasy. Nevertheless, this surface mass deficiency yields a larger geoid signal than the deep source, and the resulting geoid is a low of 14 m amplitude. A stiffer lower mantle supports the internal load more efficiently, so that the induced surface topography is smaller and yields a weaker contribution to the total geoid signal. For example, for a viscosity jump of 10 , respectively 100 , at $650 \mathrm{~km}$ depth, the surface depression reduces to $273 \mathrm{~m}$, respectively $167 \mathrm{~m}$. The total geoid is still a low of amplitude $3 \mathrm{~m}$ in the first case. It becomes a high of $17 \mathrm{~m}$ in the second case.

Second, we consider the same Earth structures but introduce two hemispheric plates. The results are indeed very different. The induced geoid and plate velocities are given in Figure 3 for a mantle viscosity contrast of 100 . The convergence is now perpendicular to the ridges and has thus

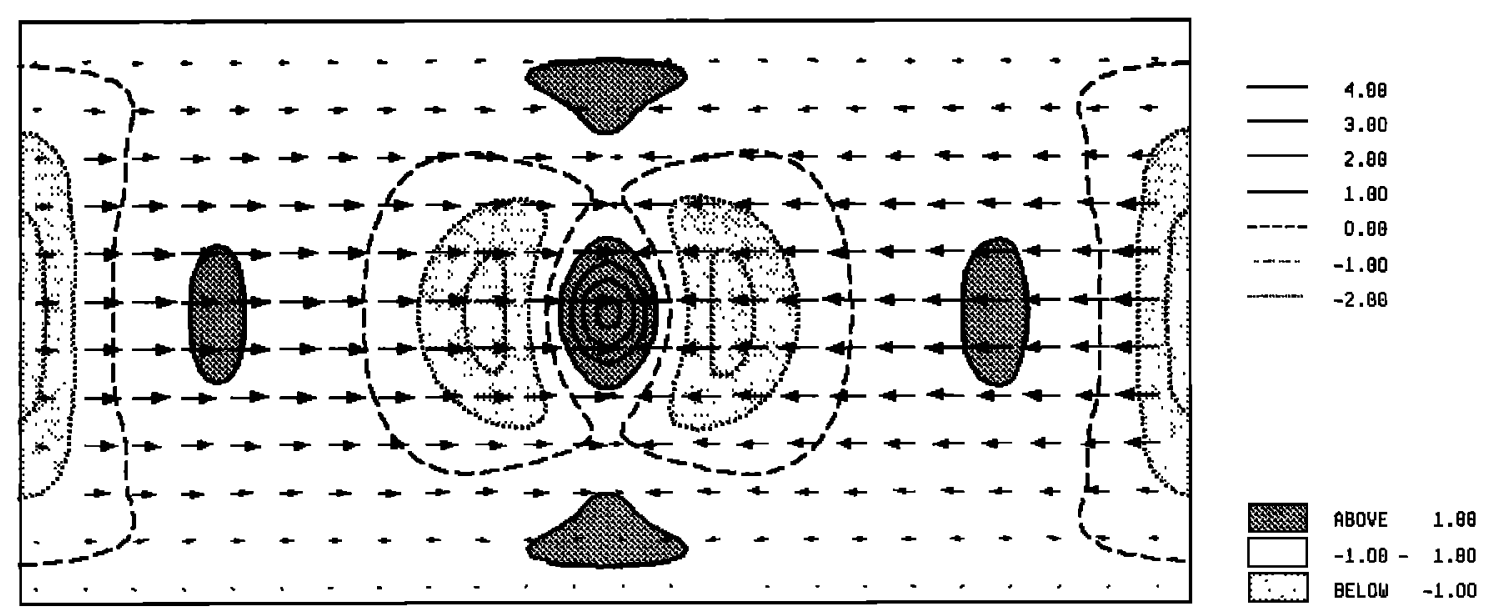

SCALE : $\rightarrow 0.06 \mathrm{CM} / \mathrm{YR}$.

Fig. 3. Computed geoid and velocity fields induced by a circular mantle mass anomaly of radius $1500 \mathrm{~km}$ located $300 \mathrm{~km}$ below the border of two hemispheric shells. This border runs through the middle of the map from north to south. This figure should be compared with Figure 1. The level lines for the geoid are $1 \mathrm{~m}$ apart and the regions above $1 \mathrm{~m}$, respectively below $-1 \mathrm{~m}$, are shaded in dark, respectively in light. The plate motions correspond to two opposite rotations around the polar axes with an equatorial velocity of $0.06 \mathrm{~cm} / \mathrm{yr}$. 
lost the symmetry of the load. The velocity is maximum at the equator and vanishes at the poles. The geoid is more complex then the one depicted in Figure 1. It superimposes a new effect related to the induced plate tectonics on the gravity signal linked to the deep mass. An overpressure exists underneath the converging zones and a pressure deficit below the diverging zones. This horizontal pressure gradient drives the return flow which now has a geometry imposed by the plate configuration. It generates an additional geoid high over trenches and a geoid low over ridges [Schubert et al., 1978; Ricard et al.. 1984]. In Figure 3, the surface velocities and the geoid are symmetrical with respect to the great circle following the plate boundary. This is, of course, related to the particular location of the mass, just beneath this circle. The same mass, located below the center of one of the hemispheric shells, would generate no surface motion and the same geoid signature as if a noslip condition was applied. Figure 4 displays the horizontal velocity and the geoid along the equator, for the three chosen viscosity stratifications. The velocity is quite uniform on each plate, and it increases again as the lower mantle viscosity decreases. The absolute value of 0.25 $\mathrm{cm} / \mathrm{yr}$ is reached for a uniform mantle. This value
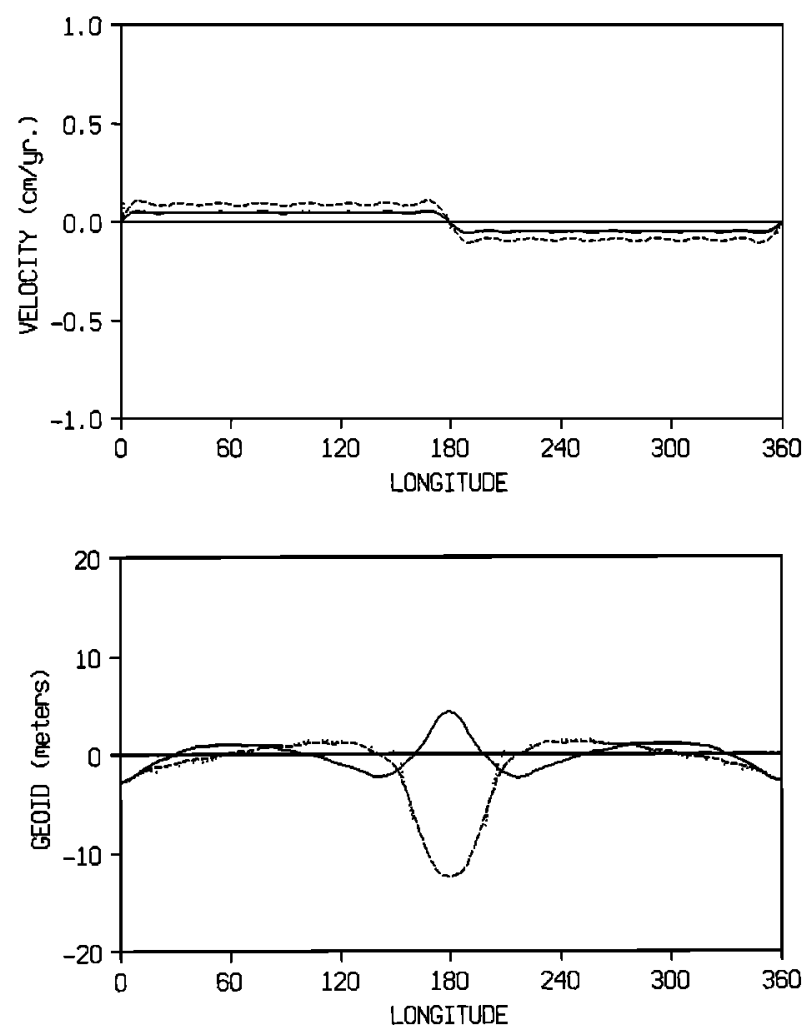

Fig. 4. Equatorial profiles of the geoid and the velocity for a model Earth with two hemispheric shells. The solid, dashed, and dotted curves correspond to a lower mantle viscosity 100,10, and 1 times that of the upper mantle, respectively. The upper mantle viscosity remains equal to $10^{21} \mathrm{~Pa} \mathrm{~s}$. This figure should be compared with Figure 2, where the profiles, drawn at the same scale, were obtained for a model Earth without plates. is 4 times lower than that obtained for a free slip model. This is because the plate, driven by the mantle flow near the load, has to drag the underlying mantle over most of the Earth's surface. In this simple example, the shape of the velocity pattern is independent of the viscosity profile which only imposes the overall amplitude. The induced topography is made of a long-wavelength component determined by the plate geometry and a local component just above the load. The resulting geoid shows these two contributions and again changes sign above the internal load when the viscosity ratio between upper and lower mantle varies from 1 to 100. The more the viscosity stratification confines the return flow, the more the computed geoids with and without plates differ.

Let us now turn to the problem of the energy distribution between the poloidal and toroidal motions. Figure 5 depicts the power spectra of the two components as a function of $l$. On top is plotted the curve corresponding to the power spectrum of the velocity field for the Earth model without plate given in Figure 1. This graph reminds us that no toroidal field can be generated in this kind of model. The increase for degrees $13-15$ is due to side lobes in the power spectrum linked with the geometry of the deep mass heterogeneity. The bottom curves are for an Earth model with plates and are associated with the velocity field depicted in Figure 3. The symmetries of the pattern requires poloidal, respectively toroidal, components of even, respectively odd degrees $l$ to be zero. Even for this very simple example a comparable energy is found in the two modes. The power spectrum of the poloidal, respectively toroidal, surface field varies as $0.02 i^{-1.8}$, respectively $0.06 i^{-2.9}$. The rotation of two hemispheric shells toward each other creates a strong vorticity in the vicinity of the two poles.

Does the existence of plates necessarily leads to an equipartition of kinetic energy between poloidal and toroidal components of the velocity field? In the case of two hemispheric plates with rotation poles located at the center of each plate, one would have a situation where only the toroidal field is present. However, this sort of hemispheric twist cannot be excited by internal loads. For a given plate configuration, the distribution of energy is certainly sensitive to the configuration of the rotational poles. In the simple case discussed above, any internal load distribution will locate the rotational axes on the plate boundary and lead to equipartion between the two velocity components at the surface.

Application to the Real Earth

In the previous section, we described the basic physics of global mantle circulation with induced plate tectonics. In what follows, let us apply our code to a realistic Earth model. To run our program, we need three different data sets: first, the number and the shapes of plates which define the coupling between the surface and the mantle; second, the three-dimensional distribution of internal masses which drive the flow; and third, the mechanical structure of the Earth characterized by viscosity and density profiles. 

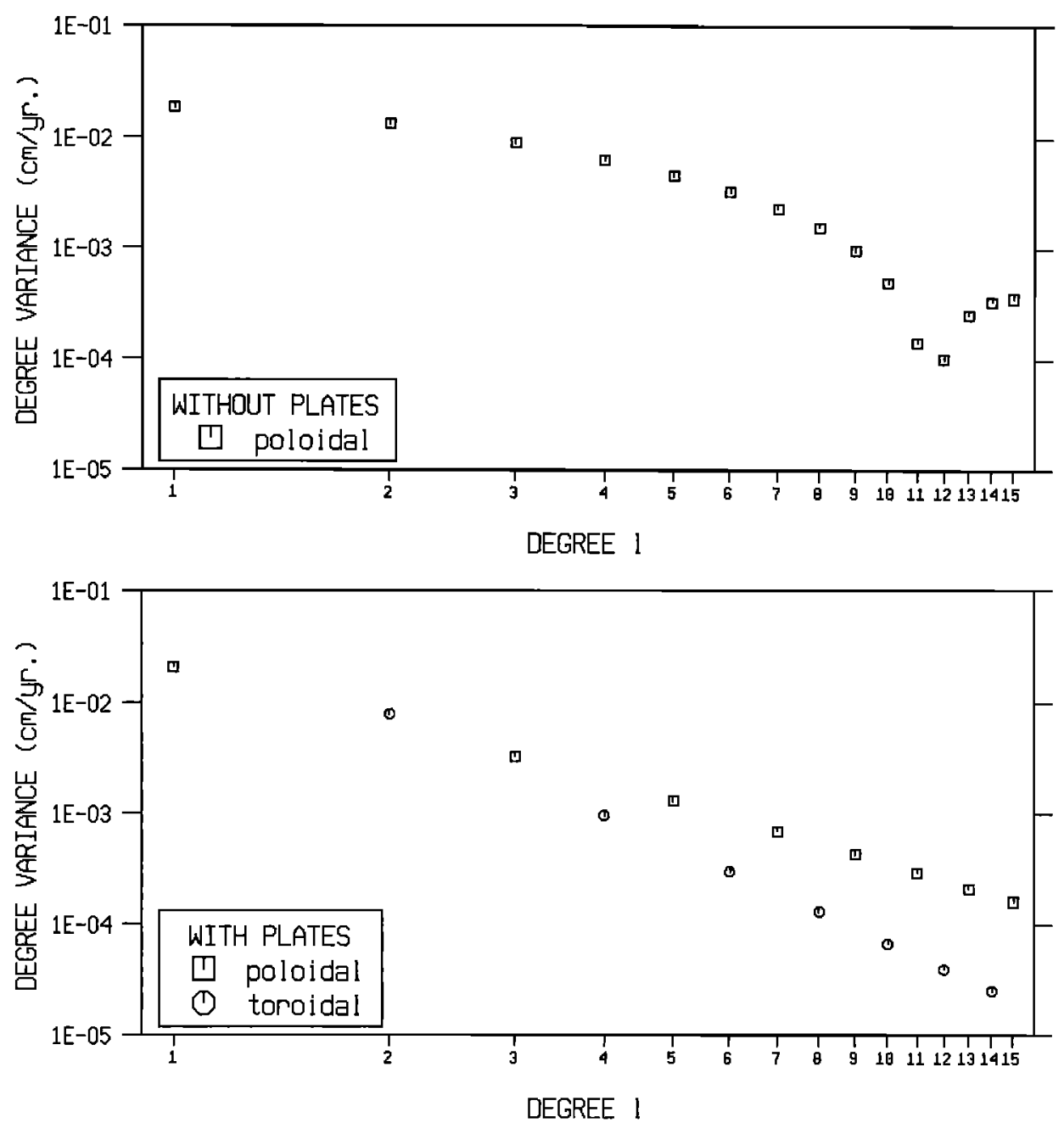

Fig. 5. Power spectra of the velocity fields depicted in Figure 1 (top) and Figure 3 (bottom). The upper graph corresponds to a model Earth with a uniform lithosphere. This assumption imposes a zero toroidal field. A lithosphere broken into two plates excites a toroidal field as seen on the lower graph. In this case, the kinematic energies of both the toroidal (circles) and poloidal (squares) motions are somewhat comparable.

We have chosen to take 11 main plates into account: African, American, Antartic, Arabian, Caribean, Cocos, Eurasian, Indian, Nazca, Pacific, and Philippine. In the lower mantle, the mass heterogeneities are supposed to be proportional to the seismic velocity anomalies revealed by tomography [Dziewonski, 1984]. A scaling factor $\partial v_{p} / \partial \rho=6 \mathrm{~km} \mathrm{~s}^{-1} / \mathrm{g} \mathrm{cm}^{-3}$ has been chosen. This value is in the range of experimental measurements for oxides [Sumino and Anderson, 1984] and roughly corresponds to what has been proposed on the basis of spherically symmetric models fitting the observed geoid [Hager et al., 1985; Ricard et al., 1989]. For the upper mantle we add two different data sets describing mass heterogeneities. The first one is proportional to upper mantle seismic tomography [Woodhouse and Dziewonski, 1984] with a conversion factor $\partial v_{s} / \partial \rho=30 \mathrm{~km} \mathrm{~s}^{-1} / \mathrm{g} \mathrm{cm}^{-3}$. This high value tends to damp the contributions of seismic heterogeneities which may, in part, have a petrological rather than thermal origin. The second one describes the excess mass associated with slabs de- fined by their seismicity [Hager, 1984]. The downgoing slabs are assumed to have a surface density of $10^{7} \mathrm{~kg} / \mathrm{m}^{2}$. This last value can be deduced from a seafloor bathymetry increase of 4000 m between young and old oceans. Other choices of this three scaling factors could have been made and will be discussed later. The various components of our mass heterogeneities are expressed in spherical harmonics truncated beyond $l=6$ for the lower mantle, beyond $l=8$ for the upper mantle, and beyond $l=15$ for the slabs. The radial properties of our model Earth are characterized by a density profile according to the Preliminary Reference Earth Model (PREM) [Dziewonski and Anderson, 1981] and a viscosity increase by a factor 50 at $650 \mathrm{~km}$. A reference value for the viscosity is chosen afterward to match the amplitudes of the predicted surface velocity field to the observed one.

For comparison we carried out two computations with the same mass heterogeneities but different lithospheric layers. In the first, the lithosphere is made of a uniform shell with viscosity 
100 times that of the upper mantle. In the second, we break the lithosphere into 11 rigid plates. In both cases, we compute the global dynamic response of our model Earth and, particularly, the three surface observables which are the poloidal and toroidal fields and the geoid. As we do not choose any absolute reference frame for the velocity description, our model with plates is unable to predict a toroidal motion of degree $l=1$, which corresponds to a global rotation of the Earth. This relative motion between the deep mantle and the plates, however, exists [Solomon et al., 1975]. The toroidal field will, of course, be zero for the model with a uniform lithosphere.

Figure 6 displays the correlation coefficients for each degree $l$, between predictions and observations, both for the surface horizontal divergence (top) and the geoid (bottom) in the case of the model Earth without plates. For the plate divergence, the meaningful correlations for degrees $l=2,4$, and 5 are mainly related to upper mantle sources. For the geoid, only the degrees $z=2$ and 3 are significantly correlated. It is wel1 known that the lower mantle heterogeneities deduced from seismic tomography explain this last observation. This correlation does not give any indication about the predicted amplitudes. The term which could induce the appropriate polar flattening of the geoid is in fact too week in the tomography we used here. Other authors [Richards and Hager, 1985] using unpublished tomography data [Clayton and Comer, 1983] found much better agreement.
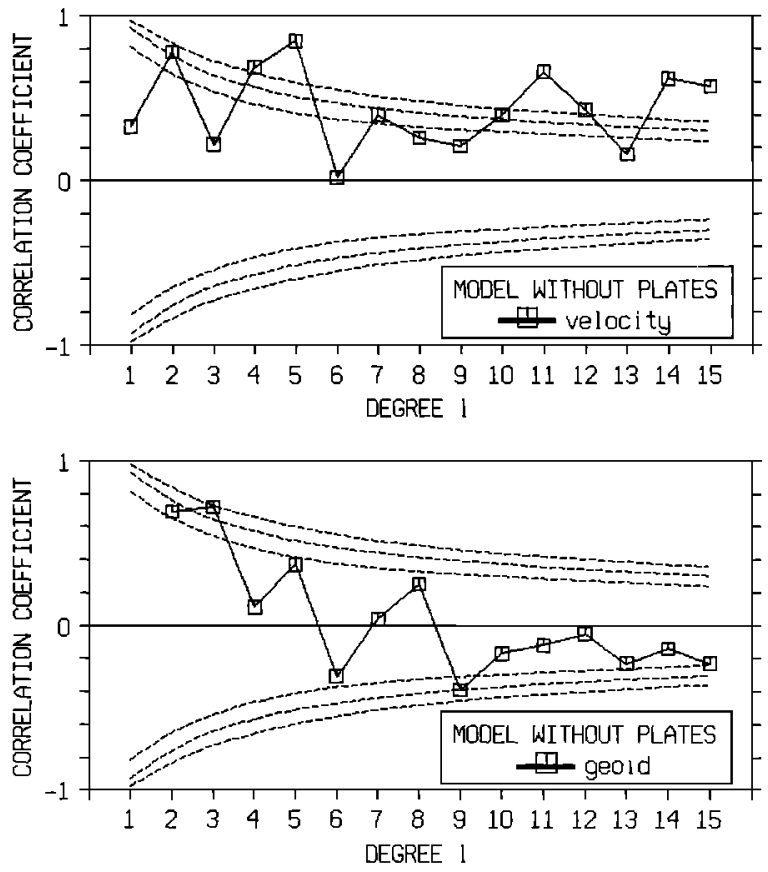

Fig. 6. Correlation coefficients for each degree $\imath$ between observations and predictions for the poloidal component of the velocity field (top) and the geoid (bottom). The case shown is for purely radial viscosity structure of an Earth model, which is loaded with density heterogeneities defined by seismic tomography and by slab seismicity. The three pairs of dashed curves represent confidence levels of 80,90 , and $95 \%$.
Figure 7 depicts the same correlation curves, but for the model Earth with 11 plates. In comparison to Figure 6 there is a great improvement. Both poloidal and toroidal velocity components are satisfactorilly predicted; 28 out of 29 coefficients are above the $90 \%$ confidence level. The geoid is also improved but less strikingly. The correlation breaks for degrees larger than 5, but the rapid decrease of the geoid spectrum with $l$ makes the absolute contributions of these large degrees very small. One must also remember that our mass heterogeneity model misses lower mantle contributions for degrees higher than 6 and upper mantle contributions related to tomography for degrees higher than 8 .

The power spectra of the computed surface velocity field split into its poloidal and toroidal components are depicted at the bottom of Figure 8. The curves for the observed velocities are plotted on the top for comparison. The general trend and amplitude of the predicted powers are highly satisfactory. We have chosen a reference value for the upper mantle viscosity of $2.6 \times 10^{20} \mathrm{~Pa} \mathrm{~s}$ in order to make the amplitudes of real and modelled spectra comparable. This yields a lower mantle viscosity of $1.3 \times 10^{22} \mathrm{~Pa} \mathrm{~s}$.

Figures 9 and 10 show the comparison between the observed (top) and computed (bottom) horizontal divergence and vertical vorticity, respectively. The existing plate motions are based on the global tectonics model of Minster and Jordan
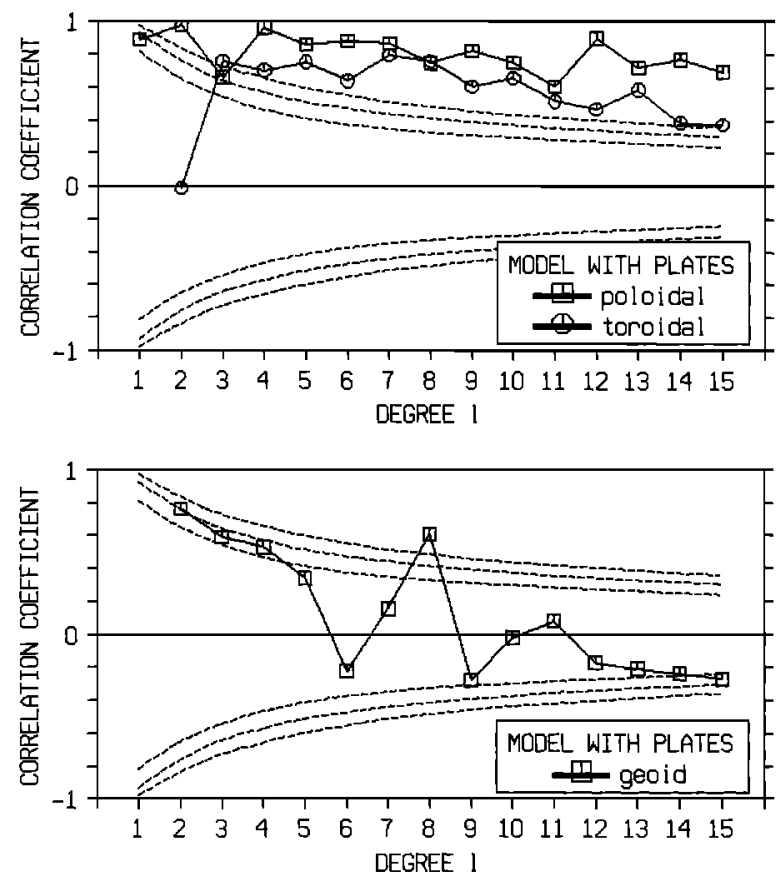

Fig. 7. Correlation coefficients between observations and predictions for the velocity field (top) and the geoid (bottom) when the 11 tectonic plates are taken into account. By comparison with Figure 6 one sees the striking increase of the correlations, both for the lower degrees of the geoid (bottom) and for all degrees of the poloidal field (top, squares). Except for degree $l=2$, the prediction of the toroidal field (top, circles) also shows a very good correlation with observations. 

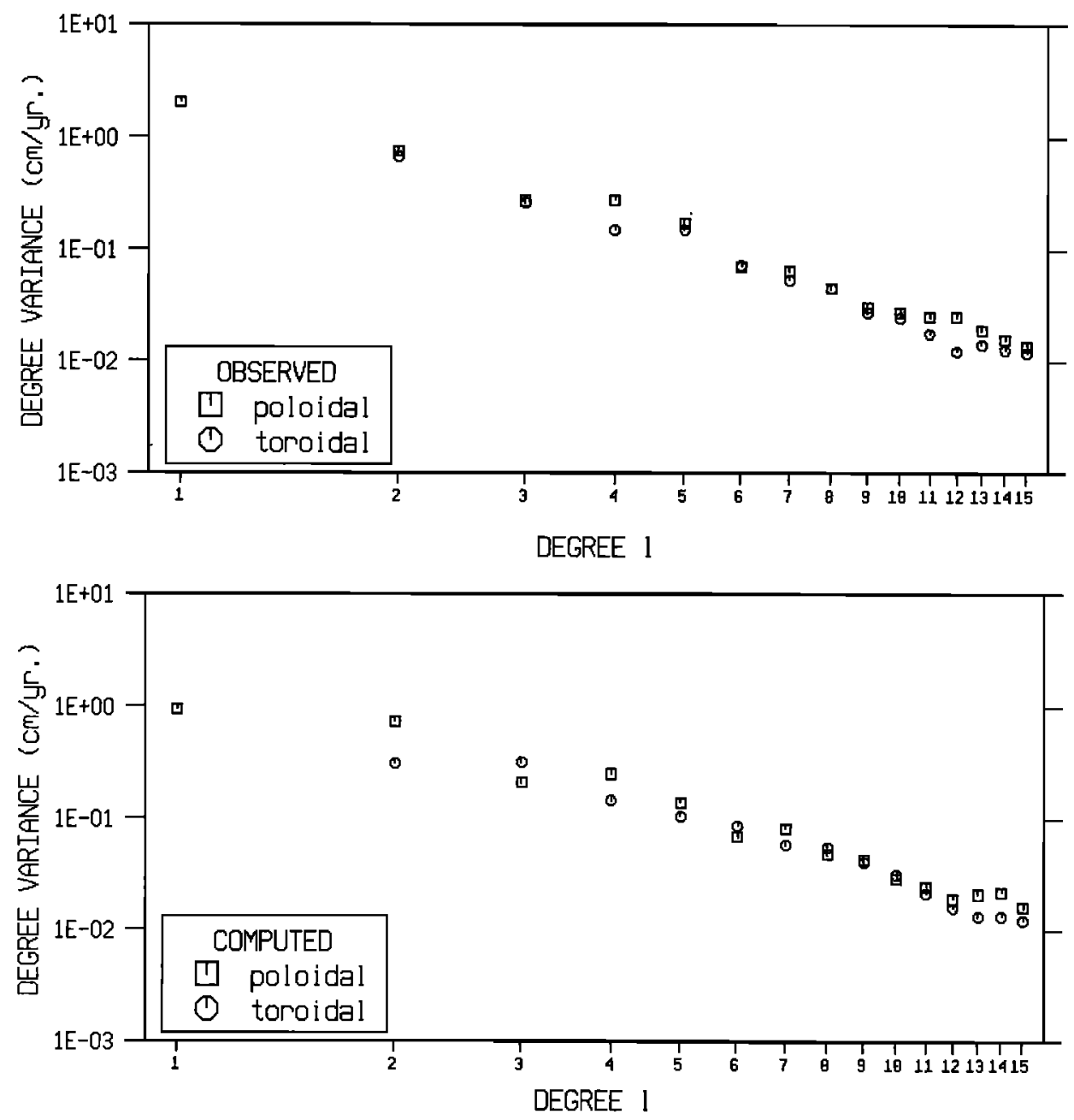

Fig. 8. The top graph depicts the power spectra of the Earth's toroidal (circle) and poloidal (squares) velocity fields derived from plate tectonics. This graph illustrates the long recognized quasi-equipartition of the observed plate motion between the two modes. The prediction of our model is depicted in the bottom graph in the same units $(\mathrm{cm} / \mathrm{yr})$. The general trend is satisfactorily predicted. The toroidal components of degree $\imath=1$ corresponding to the choice of an absolute reference frame for the velocity description have not been considered.

[1978] and are reconstructed up to degree $\ell=15$ using a previously published spectral decomposition [Forte and Peltier, 1987]. The converging zones (Figure 9, top) around the Pacific and above the Himalayan belt are depicted as light, whereas the fast spreading Nazca and East Indian ridges are dark. Our predictions (Figure 9, bottom) generally agree with observations. Nevertheless an excessive opening of the North Indian and Red Sea ridges is induced by $a$ very strong low-velocity anomaly given by the upper mantle tomographic model [Woodhouse and Dziewonski, 1984]. Our simulation also underestimates the Aleoutian subduction zone. For the observed vertical vorticity reconstructed without the degree $l=1$, which is not predictable by our model (Figure 10, top), the right, respectively left, lateral shears are shaded in dark, respectively light. The major shear zone North of Australia is correctly localized, but the strike-slip zone in California is not found by our prediction (Figure 10, bottom).
In the previous figures we have shown the two components of the surface motion separately in order to emphasize the role of the existing plates in the generation of toroidal flow. However, a direct comparison with the well-known observed motions is obviously the best way to appreciate the fit. This is depicted in Figure 11. On top, the plate motions with harmonics larger than 15 removed are described in a reference frame where the toroidal motion of degree $\imath=1$ is zero. At the bottom, our surface velocity prediction is shown. There is a good resemblance between the two. The major discrepancy comes from the position of the Pacific rotation pole which is predicted too close to California. This misfit was also revealed by the unrealistic zero vorticity near the San Andreas fault zone (Figure 10, bottom).

Let us now turn to the geoid (Figure 12). The location and amplitude of the main observed nonhydrostatic geoid features (top) are present in our prediction (bottom); particularly, the three 

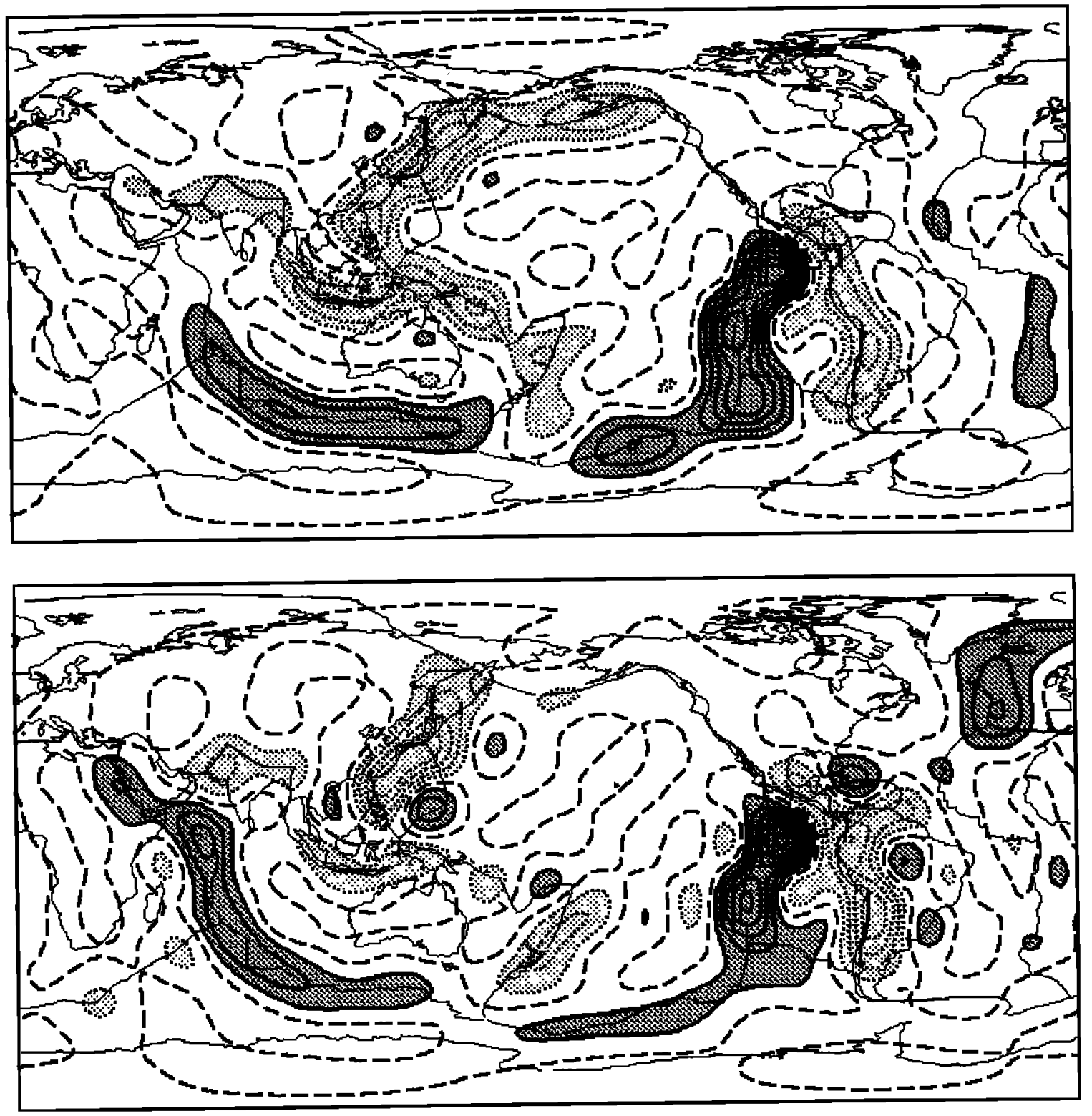

Fig. 9. Observed (top) and predicted (bottom) divergence of the surface velocity field. The description is truncated beyond degree $l=15$ of the spherical harmonics. In both maps, the level lines are $20 \times 10^{9} \mathrm{rad} / \mathrm{yr}$ apart. The maxima are shaded in dark and the minima in light. Despite some discrepancies, the global patterns are very similar.

existing geoid maxima over Africa, West Pacific, and Andes. The quality of the fit is comparable to what has been obtained for a more complex viscosity stratification and appropriate density anomalies but without plates [Ricard et al., 1989]. In terms of root-mean-square, we only explain $22 \%$ of the observed signal for degrees $l$ between 2 and 15 . The main shortcomings are the insufficient polar flattening and the presence of a strong minimum over the Nazca ridge. This spurious geoid low over the East Pacific is related to a too stong return flow induced by the opening of the ridge. The polar flattening is mainly described by the spherical harmonic of degree $l=2$ and order $m=0$. Unfortunately, as already stated, the mass anomalies with the same degree and order have a very low amplitude in the tomographic data

Another way to depict the fit between observations and predictions is to plot the harmonic coefficients of the computed fields as a function of the real ones. This is done in Figure 13: the top for the divergence and vorticity together and the bottom for the geoid. A perfect prediction would correspond to all the points lying on the diagonal of each graph. A reasonable fit is attained, with noise of about $74 \%$ of the signal for the velocities and $78 \%$ for the geoid. Taking into account the simple assumptions of the model and the uncertainties that affect the tomography, this correlation test seems to be fulfilled. Of course, one notices the poor prediction of the 

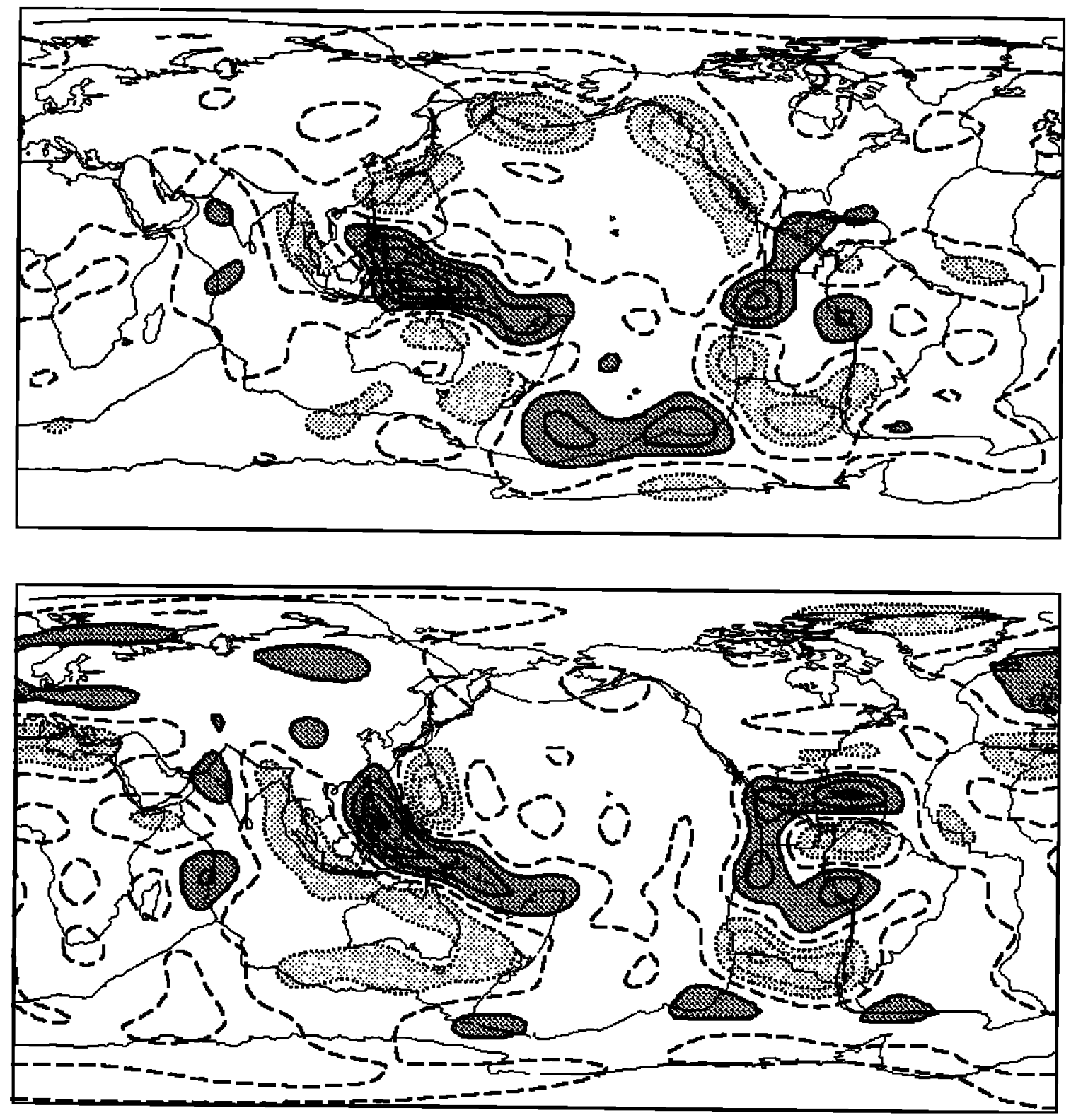

Fig. 10. Same as Figure 9, but for the vorticity. The level lines are again $20 \times 10^{9}$ $\mathrm{rad} / \mathrm{yr}$ apart. The sinistral shears are shaded in dark and the dextral ones in light. The amplitude and sign of shears between India-Australia and Pacific or between Nazca and Antarctica are well predicted. On the contrary, we miss the San Andreas shear zone. Nevertheless, the correlations between the two maps are very significant, as was quantitatively shown in Figure 7. 

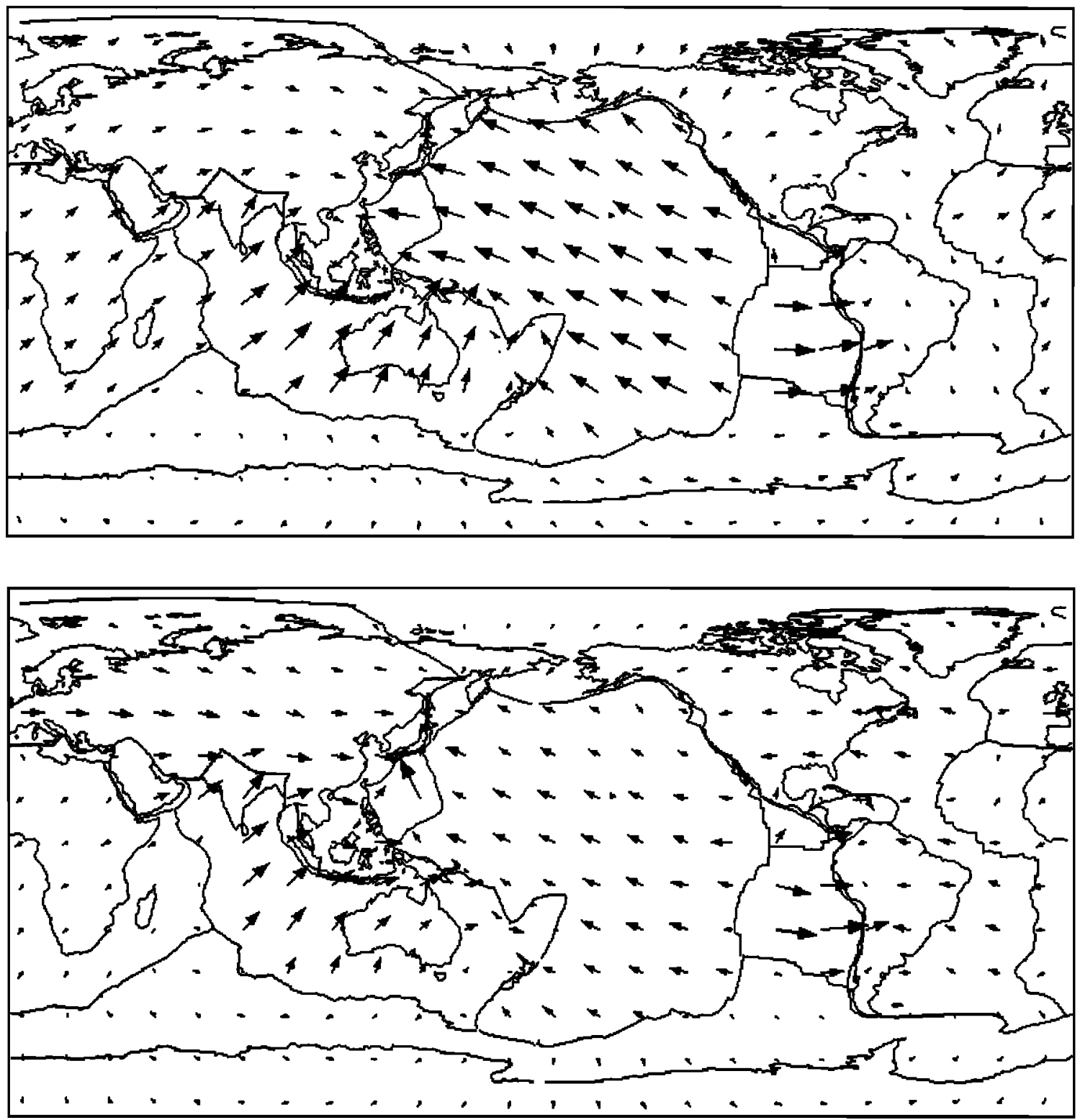

Fig. 11. The map at the top depicts the well-known Earth's plate motion filtered out for degrees $l>15$, the continental shore lines, and the plate boundaries. The map at the bottom is the result of our simulation at the same scale. The horizontal divergence and radial vorticity plotted on Figures 8 and 9 are associated with these two maps. In the usual hot spot frame, a rotation component would have been added which would have increased the Pacific motion and reduced the Eurasian and African motions. 

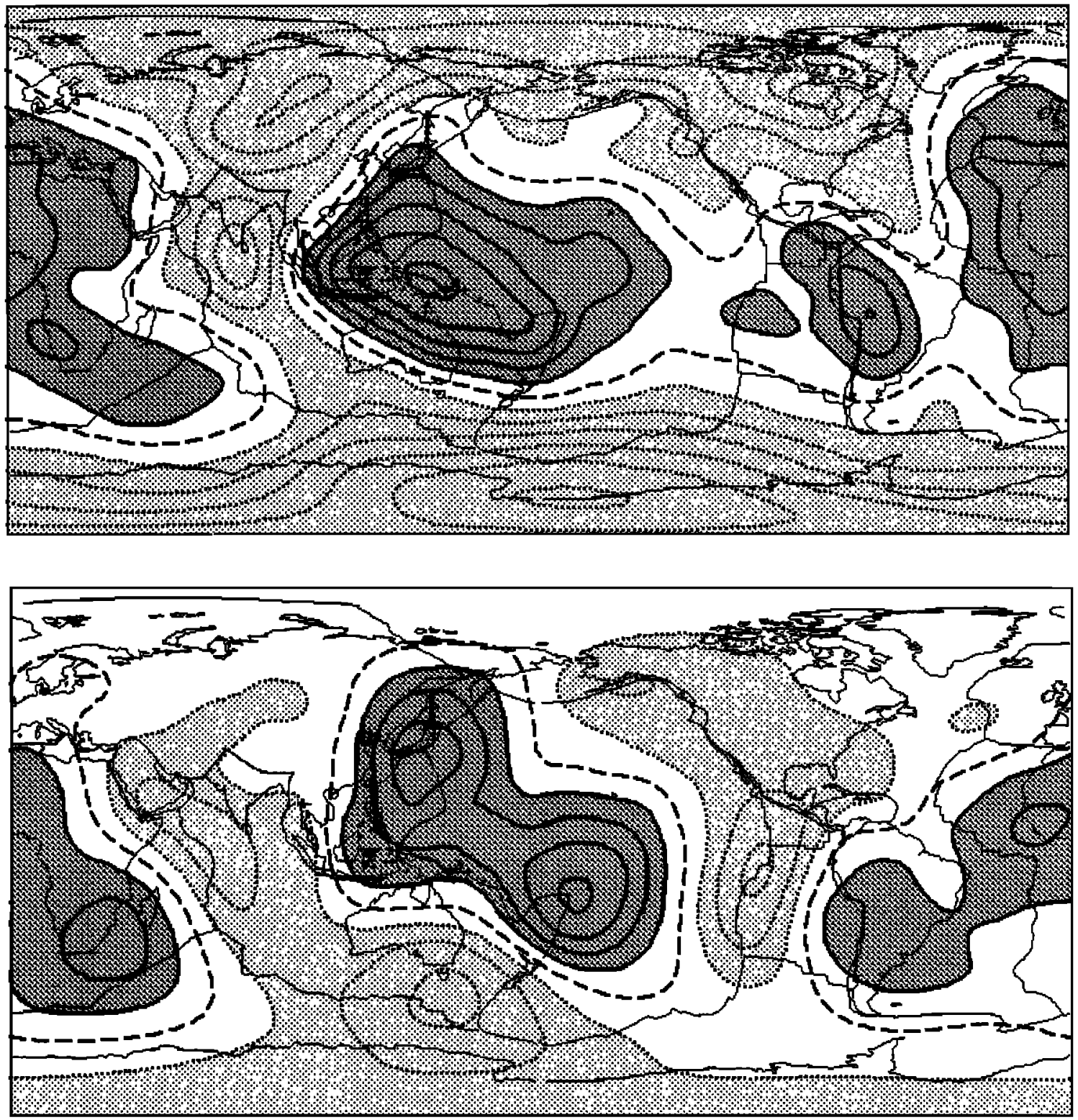

Fig. 12. Observed (top) and predicted (bottom) geoid. The level lines are $20 \mathrm{~m}$ apart. Mexima and minima are again shaded in dark and light. Our model localizes the major features, but the fit is not as good as that for the velocities. 

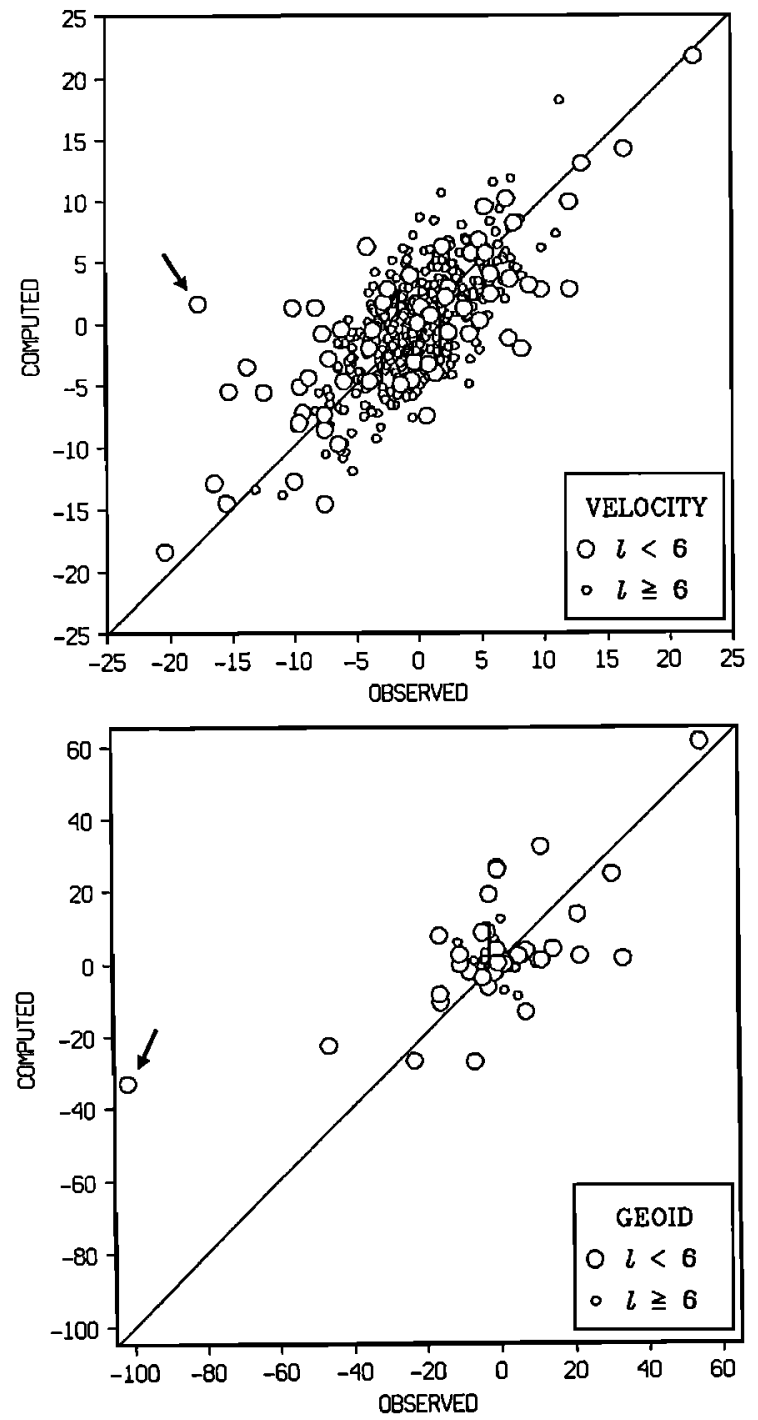

Fig. 13. The spectral components of the predicted fields are plotted against the observed ones. At the top, for the horizontal divergence and radial vorticity, and at the bottom, for the geoid. The scale is arbitrary. The major misfit, pointed by an arrow, comes from the toroidal term of degree 2 and order 1 which, for the real Earth, tends to increase the eastward migration of Africa. For the geoid, the insufficient predicted flattening associated with the term of degree 2 and order 0 (arrow) causes the principle mismatch.

degree 2 velocity field and the very bad estimation of the geoid of degree $l=2$ and $m=0$ (arrows).

To verify that the model is not too dependent upon the truncation degree $l=15$, we performed a numerical test taking only the 8 first degrees of our mass anomalies. After computing the rotation vectors of the 11 plates, the derived plate motions are expressed in spherical harmonics up to degree $l=15$. The obtained correlations between observed and predicted fields induced by these restricted excitation sources are plotted on Figure 14. Despite some differences with Figure 7 , the obtained coefficients are still comparable and significant.
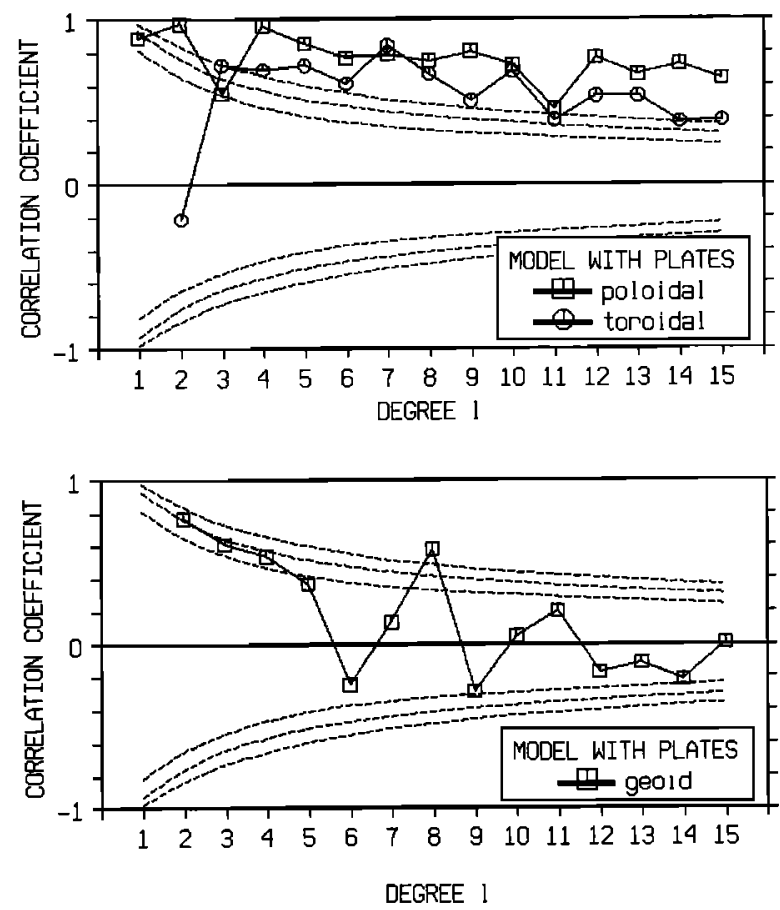

Fig. 14. Correlation coefficients between observations and predictions for the velocity (top) and the geoid (bottom). Same as Figure 7 but for mass anomalies restricted to degrees lower than 8. Notice that the correlation level remains of the same quality.

\section{Conclusions}

Several papers have been published in recent years to propose a unified approach to predict surface observables like plate velocities and the geoid from mantle heterogeneities. Using only radial variations of the mechanical properties, these models are unlikely to describe realistically the interactions between plate tectonics and mantle circulation. This paper gives a simple formalism which accounts for the very existence of plates and predicts both poloidal an toroidal velocity components. It is somewhat akin to another work on convection in Cartesian geometry below rigid plates [Gable et al.. 1988].

Using simple assumptions for the radial viscosity of the Earth and for internal mass heterogeneities, a satisfactory prediction is obtained for the surface velocities and the geoid. We consider only four parameters, the viscosity jump at $650 \mathrm{~km}$ and the three density scaling factors, one related to slabs, the other two related to upper and lower mantle tomography. Of course, the chosen values for these parameters are questionable but lead to reasonable absolute values for the upper and lower mantle viscosity. Our goal has been mainly to emphasize the role of the plates rather than to discuss or refine these values. However, we can briefly comment on the dependence of our solutions on these parameters. A more homogeneous mantle viscosity would reduce the geoid component due to plate motion but keep the velocities rather unchanged. The geoid would thus be very similar to the one obtained for a classical model with spherical symmetry and a uniform 1ithosphere. Our models are excited by 

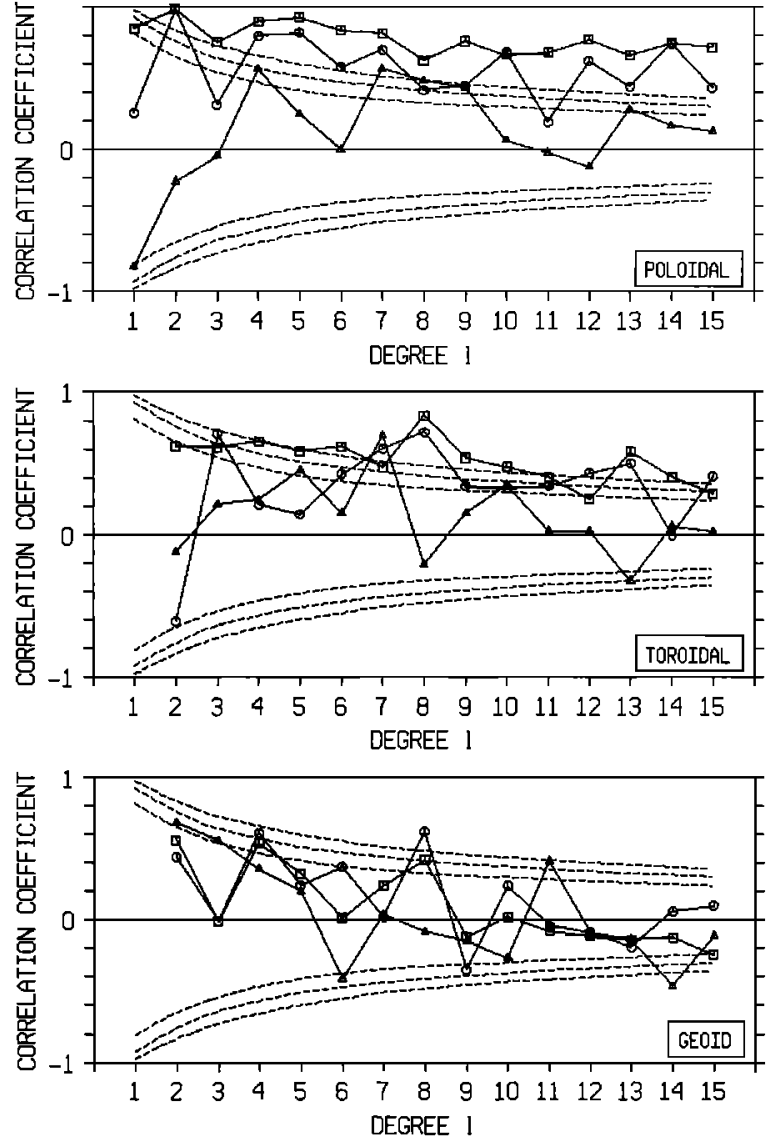

Fig. 15. Correlation coefficients between observed and computed poloidal field (top), toroidal field (middle), and geoid (bottom). The contribution of the mantle density sources have been split into three components, the slabs (squares), the upper mantle tomography (circles), and the lower mantle tomography (triangles). The slabs are the most suitable density sources to predict the two velocity components. The upper mantle tomography is also satisfactory in explaining the velocity, particularly the poloidal field. Lower mantle sources are needed to match the two first degrees of the geoid.

three distinct density sources. Their contributions to the induced surface velocity field and to the geoid can be computed separately. The correlations between these predicted contributions and the observed data set are plotted in Figure 15: top for the poloidal motion, middle for the toroidal motion and bottom for the geoid. The slabs (squares) and the upper mantle tomography (circles) lead to statisfactory correlations for the poloidal field and, by a narrow margin, for the toroidal one. The lower mantle source (triangles), on the contrary, are needed to explain the degrees 2 and 3 of the geoid but are totally inefficient in driving the plates in the right direction. By varying the relative contributions of the three sources, one can adjust the global responses of the model. Strong upper mantle sources improve the velocity but degrade the geoid prediction. The opposite is true for lower mantle sources. Such a disymmetry points to a possible decoupling between upper and lower mantle circulation.

Our results can be compared with those produced by trying to explain the plate motions by driving forces at the plate boundaries [Forsyth and Uyeda, 1975]. On the one hand, we cannot really describe the contact between plates. On the other hand, we can take into account their interactions by means of the induced mantle flow and only need a reduced number of parameters. A description of the boundary forces would be more realistic. However, we find that by taking into account the uncertainties and the smoothness of the tomographic description, our results are promising and illustrate the importance of considering the interaction between plates and mantle in global circulation models.

\section{Mathematical Appendix}

\section{General Formalism}

We simultaneously use three different vector representations. The three vector components on spherical coordinates $u_{r}, u_{\theta}, u_{p}$ are summarized as $u_{i}$. Following the notation of Phinney and Burridge [1973], we also introduce the contravariant canonical components $\mathrm{u}^{-}, \mathrm{u}^{\circ}, \mathrm{u}^{+}$given by

$$
\begin{aligned}
& u^{-}=\frac{1}{\sqrt{2}}\left(u_{\theta}+i u_{\varphi}\right) \\
& u^{0}=u_{r} \\
& u^{+}=\frac{1}{\sqrt{2}}\left(-u_{\theta}+i u_{\varphi}\right)
\end{aligned}
$$

These components are abbreviated as $u^{n}$ where $n$ stands for,+ 0 or -. Finally, we also need a vector description in terms of generalized spherical harmonics. These functions are defined by

$$
Y_{l m}^{n}=P_{l m}^{n}(\cos \theta) i m \varphi
$$

where $P_{l m}^{n}(\cos \theta)$ is a generalized Legendre function as defined by Phinney and Burridge [1973]. For $n=0$ one has the ordinary pherical harmonics. The vector components on such a basis are written $u^{\text {nlm }}$, where $l$ and $m$ are the harmonic degree and order, respectively. The interest of this formalism has been strongly advocated by seismologists [Woodhouse, 1980; Mochizuki, 1986; Tanimoto, 1986]. It provides an elegant way of writing differential operations such as gradient, divergence, or curl.

The direct and inverse transformations between these spatial, complex, and spectral spaces are easily expressed. A simple matrix $\mathrm{C}$ and its Hermitian conjugate $C^{*}$ allow the transformations of spatial to contravariant components. We write these relationships in the form

$$
\begin{aligned}
& u^{n}=c_{n 1}^{*} u_{1} \\
& u_{1}=c_{n i} u^{n}
\end{aligned}
$$

Direct and inverse discrete transformations between $u^{n}$ and $u^{n} l m$ can be defined [Lognonné and Romanowicz, 1989], they read 


$$
\begin{aligned}
u^{n l m} & =\sum_{\theta} \sum_{\varphi} u^{n} w(\theta) P_{l m}^{n}(\cos \theta) e^{-i m \varphi} \\
u^{n} & =\sum_{l} \sum_{m} u^{n l m} P_{l m}^{n}(\cos \theta) e^{i m \varphi}
\end{aligned}
$$

In the first expression, the integration in longitude is carried out by a fast Fourier transform, whereas the latitude integration is handled by a Gaussian quadrature with weights $w(\theta)$. The spatial grid is thus expressed by $2 \times l_{\max }$ equally spaced Fourier points in longitude $\varphi$ and $2 \times l_{\text {ma }}$ Gauss points inequally spaced in latitude $\theta$. We simplify these equations by means of the matrix $M$ such as

$$
\begin{aligned}
u^{n l m} & =\left(w(\theta) M_{l m}\right)^{*} u^{n} \\
u^{n} & =\left(M_{l m}\right) u^{n l m}
\end{aligned}
$$

The use of generalized harmonics representation is very convenient to express the horizontal divergence and the vertical vorticity. They are easily deduced on the ordinary spherical harmonic basis from the following equations:

$$
\begin{gathered}
\left(\vec{\nabla}_{2} \overrightarrow{\mathrm{v}}\right)^{0 l m}=\frac{\mathrm{u}^{+l m}+\mathrm{u}^{-l m}}{\mathrm{R}} \sqrt{\frac{l(l+1)}{2}} \\
\left((\vec{\nabla} \otimes \overrightarrow{\mathrm{v}}) \overrightarrow{\mathrm{e}}_{1}\right)^{0 l m}=\frac{\mathrm{u}^{+l m}-\mathrm{u}^{-l m}}{\mathrm{R}} \mathrm{i} \sqrt{\frac{l(l+1)}{2}}
\end{gathered}
$$

In the last equation $\vec{e}_{1}$ is the radial unit vector.

\section{Dynamics of the Viscous Mantle}

The case of a purely radial viscosity structure has been solved on the basis of the NavierStokes equation for an incompressible fluid with an infinite Prandtl number [Kaula, 1975; Hager and $0^{\prime}$ Connell, 1979; Ricard et al., 1984]. Here we only summarize the main results of these studies. The stresses induced beneath a rigid lithosphere by three-dimensional mantle heterogeneities $\delta m(r, \theta, \varphi)$ are computed using a propagator which depends on the viscosity and on the density radial profiles of the mantle. Their expressions in terms of generalized harmonic components are abbreviated to $\tau_{i n t}^{n} l m$. In the absence of internal loads, the surface velocity with components $v^{n i m}$ also generates a stress field $\tau^{n} l m$. This stress field is deduced from the velocity by a linear operator $K$, function of the radial mechanical parameters:

$$
\tau_{e \times t}^{n l m}=\frac{\eta_{0}}{R} K v^{n} \cdot l \cdot m \cdot
$$

We introduce a reference viscosity $\eta_{0}$ and the Earth's radius $R$ to insure the nondimensionality of the matrix $\mathrm{K}$. The computation of other surface observables, like topography or gravity, induced by internal loads and/or imposed surface velocities has also been described in the previously quoted papers.

\section{Plate Dynamics}

The kinematics of $j$ plates on a spherical Earth imposes the same number of rotation vectors $\vec{\Omega}_{j}$. Thus the surface velocity reads

$$
u_{i}=\sum_{j}\left(\vec{\Omega}_{j} \otimes R \vec{e}_{1}\right) \vec{e}_{i} \delta(j, \theta \varphi)
$$

where $\delta(j, \theta \varphi)$ is 1 or 0 depending on whether the coordinates $\theta$ and $\varphi$ point to the jth plate or not, $\vec{e}_{1}$ represent the basic vectors on spherical coordinates. Let us write $\Omega_{\mathrm{jk}}$ the vector components of each rotation vector on a Cartesian basis with vectors $\vec{E}_{k}$. Equation (A8) can then be rearranged to

$$
u_{i}=\sum_{j k} R\left(\vec{e}_{1} \otimes \vec{e}_{i}\right) \vec{E}_{k} \delta(j, \theta \varphi) \Omega_{j k}
$$

which, written in a compact form, reads

$$
u_{i}=R\left(P_{j k}^{1}\right)^{*} \Omega_{j k}
$$

We calculate the total torque $\vec{m}_{j}$ on each plate $j$ using the Gaussian quadrature with weights $w(\theta)$, assuming that the only force acting on the plate is the underlying drag $\vec{\tau}$ :

$$
\overrightarrow{\mathrm{m}}_{j}=\sum_{\theta} \sum_{\varphi} R\left(\overrightarrow{\mathrm{e}}_{1} \otimes \overrightarrow{\mathrm{T}}\right) \delta(j, \theta \varphi) R^{2} w(\theta)
$$

Projecting (A12) onto the Cartesian basis with vector $\overrightarrow{\mathrm{E}}_{\mathbf{k}}$ and permutating its terms, it reads

$m_{j k}=\sum_{i} \sum_{\theta} \sum_{\varphi} R^{3}\left(\vec{e}_{1} \otimes \vec{e}_{i}\right) \vec{E}_{k} \delta(j, \theta \varphi) \tau_{i} \sin \theta \Delta \theta \Delta \varphi$

This yields

$$
m_{j k}=R^{3}\left(w(\theta) P_{j k}^{1}\right) T_{1}
$$

\section{Plate Equilibrium}

In the absence of direct interplate forces, the plate equilibrium imposes the global mantle flow to exert a zero torque on the plates. This flow can be expressed as the superposition of two dynamic configurations. The first is driven by mantle mass heterogeneities in a model Earth with a no-slip boundary condition at the surface. The second is excited by plate motions without internal loads. When the torques imposed by the two dynamical configurations are balanced, the global solution is found.

Using (A14), (A3), and (A5) and omitting for simplicity the indices, the torques due to the first configuration are

$$
m_{j k}=R^{3} \quad(w P) C \text { (M) } T_{i n t}^{n l m}
$$

With rigid plates of vanishing thicknesses, one can neglect the vertical flow induced at plate boundaries. Using this assumption and the equations (A15), (A8), (A5), (A3), and (A11), the torques exerted by imposed plate motions are: 


$$
m_{j k}=\eta_{0} R^{3}(w P) C(M) K(w M)^{*} C^{*}(P)^{*} \Omega_{j} \cdot k \text {. }
$$

Introducing the matrix $G$ which is the product w P C M, the equations (A14) and (A15) yield

$$
\Omega_{j k}=\frac{1}{\eta_{0}}\left(G K G^{*}\right)^{-1} G \tau_{i n t}^{n l m}
$$

The rotation vectors of a model Earth with plates can therefore be deduced from the dynamics induced by internal loads acting below a model Earth with a purely rigid lithosphere. In equation (A17), G only depends upon the plate geometry whereas $K$ is a function of the viscosity and density profiles. The velocity field in terms of generalized harmonic components reads

$$
v^{n} l m=\frac{R}{\eta_{0}} G^{*}\left(G K G^{*}\right)^{-1} G \tau_{1 n t}^{n l m}
$$

When the 1ithospheric thickness $L$ is no more negligible, a new effect must be taken into account. The mass conservation associates a vertical flow to the horizontal divergence of the lithosphere. Integrating the conservation equation through the lithospheric thickness, this vertical component reads

$$
\mathrm{v}_{\mathrm{r}}=\mathrm{L} \vec{\nabla}_{\mathrm{H}} \overrightarrow{\mathrm{V}}
$$

where $\vec{\nabla}_{H}$ is the horizontal divergence. This vertical velocity also induces horizontal stresses. The surface velocity field $\left(\mathrm{u}^{-l m}, 0, \mathrm{u}^{+l m}\right)_{0}$ can be converted to the sublithospheric velocity field $\left(u^{-l m}, 0, u^{+l m}\right)$ by means of a matrix E containing the following elements:

$$
E=I d+\frac{L}{R} \sqrt{\frac{l(Z+1)}{2}}\left(\begin{array}{lll}
0 & 0 & 0 \\
1 & 0 & 1 \\
0 & 0 & 0
\end{array}\right)
$$

where Id is the identity matrix. When the lithospheric thickness $L$ vanishes, the $E$ matrix equals to the identity matrix. For a given plate thickness the vertical component increases with the degree $l$, i.e., as the wavelength decreases. In equations (A8), (A17), and (A18), $K$ must be replaced by $K E$ to mimic the effect of a finite lithospheric thickness. When the surface velocity $\mathrm{v}^{\mathrm{n} l m}$ and the internal loads $\delta \mathrm{m}(\mathrm{r}, \theta, \varphi)$ are given, all the surface observables are then computed by a classical propagator method.

Acknowledgments. This work was supported by the Institut National des Sciences de l'Univers. Contribution 99 of the INSU-"Dynamique et Bilan de la Terre" global coupling program.

References

Clayton, R. W., and R. P. Comer, A tomographic analysis of mantle heterogeneities from body waves travel times, Eos Trans. AGU, 64, 776, 1983.

Dziewonski, A. M., Mapping the lower mantle: Determination of lateral heterogeneity in $P$ velocity up to degree and order 6, J. Geophys. Res., 89, 5929-5952, 1984.
Dziewonski, A. M., and D. L. Anderson, Preliminary reference earth model (PREM), Phys. Earth Plenet. Inter, 25, 297-356, 1981.

Forsyth, D. W., and S. Uyeda, On the relative importance of the driving forces of plate motion, Geophys. J. R. Astron. Soc., 43, 163$200,1975$.

Forte, A. M., and W. R. Peltier, Plate tectonics and aspheroidal Earth structure: The import ance of poloidal-toroidal coupling, J. Geo phys. Res., 92, 3645-3679, 1987.

Gable, C. W., R. J. O'Connell, and B. J. Travis, Plate motion in models of 3-D convection with layered viscosity: Implications for mantle flow, Eos Trans. AGU, 69, 1414, 1988.

Hager, B. H., Subducted slabs and the geoid: Constraints on mantle rheology and flow, J. Geophys. Res., 89, 6003-6016, 1984.

Hager, B. H., and R. J. O'Connell, Kinematic models of large-scale flow in the Earth's mantle, J. Geophys. Res., 84, 1031-1048, 1979.

Hager, B. H., and M. A. Richards, The generation of long-wavelength geoid anomalies: Implications for mantle structure and dynamics, Terra Cognita, 4, 247, 1984.

Hager, B. H., R. W. Clayton, M. A. Richards, R. P. Comer, and A. M. Dziewonski, Lower mantle heterogeneity, dynamic topography, and the geoid, Nature, 313, 541-545, 1985.

Kaula, W. M., Absolute plate motions by boundary velocity minimization, J. Geophys. Res.. 80, 244-248, 1975.

Kaula, W. M., Material properties for mantle convection consistent with observed surface fields, J. Geophys. Res., 85, 7031-7044, 1980.

Lago, B., and M. Rabinowicz, Admittance for a convection in a layered spherical shell, Geophys. J. R. Astron. Soc., 77, 461-482, 1984.

Lliboutry, L. A., The driving mechanism, its source of energy and its evolution studied with a three-layer model, J. Geophys. Res., 77. 3759-3770, 1972.

Lognonné, P., and B. Romanowicz, Coupled Earth vibrations: The spectral method, Geophys. J., in press, 1989.

Minster, J. B., and H. Jordan, Present-day plate motions, J. Geophys. Res., 83. 5331-5354, 1978.

Mochizuki E., The free oscillations of an anisotropic and heterogeneous Earth, Geophys. J. R. Astron. Soc., 86, 167-176, 1986.

Phinney, R. A., and R. Burridge, Representation of the elastic-gravitational excitation of a spherical Earth model by generalized spherical harmonics, Geophys. J. R. Astron. Soc., 74 . $451-487,1973$.

Ricard, Y., L. Fleitout, and C. Froidevaux, Geoid heights and lithospheric stresses for a dynamic Earth, Ann. Geophys, 2. 267-286, 1984.

Ricard, Y., C. Froidevaux, and L., Fleitout, Global plate motion and the geoid: A physical model, Geophys. J., 93, 477-484, 1988.

Ricard, Y., C. Vigny, and C. Froidevaux, Mantle heterogeneities and plate motion: A Monte Carlo Inversion, J. Geophys. Res., in press, 1989.

Richards, M. A., and B. H. Hager, Geoid anomaly in a dynamic Earth, J. Geophys. Res., 89, 5987-6002, 1984.

Richards, M. A., and B. H. Hager, The Earth's geoid and the large-scale structure of mantle 
convection, Proceedings of the NATO Advance Study Institute: Physics of planets, Universi ty of Newcastle Upon Tyne, 9-20, Avril, 1985.

Richards, M. A., and B. H. Hager, Effects of lateral viscosity variations on long-wavelength geoid anomalies and topography, J. Geophys. Res., 94, 10299-10313, 1989.

Schubert, G., D. A. Yuen, C. Froidevaux, L. Fleitout, and M. Souriau, Mantle circulation with partial shallow return flow: Effects on stresses in oceanic plates and topography of the seafloor, J. Geophys. Res., 83. 745-758, 1978.

Solomon, S. C., N. H. Sleep, and R. M. Richardson, On the forces driving plate tecto nics: Inference from absolute plate velocities and intraplate stress, Geophys. J. R. Astron. Soc., 42, 769-801, 1975.

Sumino, Y., and 0. L. Anderson, Elastic constants of minerals. in Handbook of physical properties of rocks, vol. 3 , edited by $\mathrm{R}$. S. Carmichae1, pp. 39-138, CRC press, Boca Raton, Fla., 1984 .
Tanimoto, T., Free oscillations of a slightly anisotropic Earth, Geophys. J. R. Astron. Soc., 87, 493-517, 1986.

Woodhouse, J. H., The coupling and attenuation of nealy resonant multiplets in the Earth's free oscillations spectrum, Geophys. J. R. Astron. Soc., 61, 261-283, 1980 .

Woodhouse, J. H., and A. M. Dziewonski, Mapping the upper mantle: Three-dimentional modeling of Earth structure by inversion of seismic waveforms, J. Geophys. Res., 80, 1915-1919, 1984.

Y. Ricard and C. Vigny, Département de Géophysique, Ecole normale Supérieure, 24 rue Lhomond, 75231 Paris Cedex 05. France.

(Received March 14, 1989; revised June 15, 1989; accepted June 19, 1989) 